\title{
Energy Evolution Model and Critical Failure Criterion for the Dynamic Process of Tunnel System Instability
}

\author{
Zhen Liu $\cdot$ Cuiying Zhou $\cdot$ Xu Yang
}

Received: 26 June 2013/Accepted: 5 June 2014/Published online: 15 June 2014

(C) The Author(s) 2014. This article is published with open access at Springerlink.com

\begin{abstract}
The failure criteria and dynamic evolution process of tunnel system deformation and instability have been one of the popular areas in the research field of underground engineering albeit one of the most difficult. The energy evolution model and critical failure criterion of the dynamic process in tunnel system instability are studied in this paper. Based on in situ measurements and the dissipative structure theory, the energy dissipation and dynamic evolution characteristics of the tunnel system instability has been presented. By using the basic laws of thermodynamics, the energy dissipation mechanism of the whole tunnel system has been explored, and an energy evolution model of the tunnel system instability has been established. On this basis, the degree of stability evolution process has been determined depending on the sample size (more than or $<3,000$ ). The energy criterion is proposed in accordance with the unstable
\end{abstract}

Z. Liu ( $\square)$

School of Marine Sciences, Sun Yat-sen University, Guangzhou 510275, China

e-mail: liuzh8@mail.sysu.edu.cn

Z. Liu $\cdot$ C. Zhou $\cdot$ X. Yang

Research Center for Geotechnical Engineering and Information Technology, Sun Yat-sen University, Guangzhou 510275, China

e-mail: ueit@mail.sysu.edu.cn

C. Zhou $\cdot$ X. Yang

School of Engineering, Sun Yat-sen University, Guangzhou 510275, China and failure features of each evaluation stage of the tunnel system. This study has proved that the energy evolution model and critical failure criterion is reasonable and reliable in studying the stability analysis of an overlapped tunnels in Guangzhou metro, China. It also provides a significant guideline for the calculation and analysis of tunnel stability.

Keywords Tunnel system - Dynamic instability · Energy $\cdot$ Evolution model $\cdot$ Failure criterion

\section{Introduction}

Tunnel engineering is a major and integral part of transportation infrastructure. The failure criteria and evolution process of tunnel deformation and instability have been popular areas in research in underground engineering, albeit one of the most difficult. The key is to quantitatively reveal the deformation process and critical state of tunnel instability and failure. The present method of approach is mainly based on two kinds means: one which is based on the monitoring data analysis and the other on the research and criteria for deformation failure mechanisms. Both of them have their own distinguishing features and have provided progress to the field. The former is the most widely used method, which is mainly based on the analysis of monitored data and the control variable when a large number of engineering disaster events 
occur, thus determining the critical criteria of instability. Among them, the displacement monitoring is often used in discriminating the tunnel instability, including the permissible limit displacement, displacement rate, displacement acceleration and deformation rate ratio etc. (Komiya et al. 2000; Carranza Torres 2004; Bhasin Bhasin et al. 2006; Li 1998; Zhang and Liu 2003). All these establish instability and the failure critical criteria by fitting the evolving rules and model of monitoring displacement sequence. The method is more suitable for research on the critical state and lacking of the consideration in different evolution stages state of tunnel instability and failure. And the method is difficult to quantitatively describe the key process of tunnel instability and failure. The latter are still in the exploration stage, and have mainly established the critical instability and failure criteria by using the nonlinear theory and numerical calculation method (Yang and Yin 2006; Heap and Faulkner 2008; Liu et al. 2008; Cui 2010; Yang and Yang 2010; Yang and Huang 2011; Sarat 2011; Serrano et al. 2011; Huang and Yang 2011; Aydan et al. 2012). Based on this, it mainly establishes the sharp point catastrophe model and criterion by using the catastrophe theory (Liu et al. 2002; Chen 2002; Yue et al. 2006; Yan and Xu 2006; Jiancong 2008; Wenjiang et al. 2011; Hong 2013). All of the latter study have established a sharp point catastrophe unstable model and the critical mechanics by analysing tunnel deformation instability and failure mechanism. The method is limited to simplified boundary and single load model and lacking of the consideration in the interaction of various factors tunnel system evolution. However, tunnel deformation instability often results from the combination of many effects in complex environmental conditions, which is the key part of the evolution process and most difficult elements to control in terms of risk in a project.

The energy dissipation mechanism and evolution characteristics for the whole dynamic process of the tunnel system instability are analyzed by the dissipative structure theory and in situ measurements. By using the basic laws of thermodynamics, an energy evolution model of the tunnel system instability is established. This paper will provide an energy criterion of the stability of the whole process of the evolution with large and small quantities of data. Then it also puts forward the energy critical criterion of destruction to judge the stability degree of the tunnel system. Finally, it is concluded that this study provides an analytical basis for the risk control in the process of construction through an analysis of the TiyuxiTiyuzhongxin of Guangzhou metro No. 1 line. The study puts forward discriminate method in different evolutionary stages and instable critical state. It also provides new ideas and methods in tunnel engineering risk control.

\section{Basic of the Theory of Energy Evolution}

\subsection{Basic of the Theory of Dissipative Structure}

From the viewpoint of a system, the tunnel system is a complicated system of lining, surrounding rock, and other subsystems. Its deformation and instability are an irreversible energy dissipation process that is highly nonlinear and complex. Therefore, this paper employs the dissipative structure theory to study the dynamic evolution of tunnel system instability.

The dissipative structure theory concerns the evolution of an equilibrium open system from disorder to order, which mainly uses the thermodynamics and statistical physics to describe the formation process and mechanism of a dissipative structure (Ilya Prigogine 1969). The dissipative structure is a new stable macro and ordered structure, which is a complex system far from equilibrium maintained in an external energy or material flow by self-organized. Its formation of the dissipative structure must satisfy three conditions (Yang 1996; Cai 1998): an open system far from equilibrium, nonlinear interaction and the presence of fluctuations. An open system is a system that there are energy exchange and material exchange between the system and the environment. Far from equilibrium is a very uneven state of the physical properties which can be measured in a system. Nonlinear interaction takes place between different subsystems within a system. Fluctuation means the macro parameters that deviate from the average, with the advantage of the independent movement of a subsystems and the random interference of the environmental conditions. The macro parameters of deformation could be used to describe the fluctuation in tunnel system. The formation process of a dissipative structure (Cai 1998) comprises non-linear open systems far from the continuous equilibrium exchange of matter and energy with the outside world. When 
some parameter within the system reaches a certain threshold, mutation may take place in the system (nonequilibrium phase transition) by fluctuation from a chaotic state of disorder into an orderly state of time, space and function.

\subsection{Basic of Thermodynamics}

From the view of the system theory the evolution of tunnel system needs to exchange the energy with the external occurrence environment. Thermodynamics is a branch of natural science concerned with heat and its relation to energy and work. It defines macroscopic variables (such as temperature, internal energy, entropy, and pressure) that characterize materials and radiation, and explains how they are related and by what laws they change with time (Haining 2009). Thermodynamics describes the average behavior of very large numbers of microscopic constituents, and the laws of thermodynamics can be derived from statistical mechanics. Much of the empirical content of thermodynamics is contained in the four laws. The first law of thermodynamics asserts the existence of a quantity called the internal energy of a system, which is distinguishable from the kinetic energy of bulk movement of the system and from its potential energy with respect to its surroundings (Haining 2009). The first law distinguishes transfers of energy between closed systems as heat and as work. The second law of thermodynamics concerns two quantities called temperature and entropy. Entropy expresses the limitations, arising from what is known as irreversibility, on the amount of thermodynamic work that can be delivered to an external system by a thermodynamic process. Temperature, whose properties are also partially described by the zeroth law of thermodynamics, quantifies the direction of energy flow as heat between two systems in thermal contact and quantifies the common-sense notions of "hot" and "cold" (Haining 2009). There are also the third law of thermodynamics (as a system approaches absolute zero the entropy of the system approaches a minimum value) and the zeroth law of thermodynamics (if two systems are each in thermal equilibrium with a third, they are also in thermal equilibrium with each other). But the first and second law of thermodynamics are used in this study. Therefore, the energy evolution of tunnel system could be by the entropy and laws of thermodynamic.

\subsection{Basic of Information Theory}

The energy evolution of tunnel system could be described by the entropy in thermodynamics. But the entropy in thermodynamics is difficult to measure and calculate. But the entropy in the information theory is just right to solve this problem. Information theory is a branch of applied mathematics, electrical engineering, bioinformatics, and computer science involving the quantification of information (Yuheng 1989). A key measure of information is entropy, which is usually expressed by the average number of bits needed to store or communicate one symbol in a message. Entropy quantifies the uncertainty involved in predicting the value of a random variable. The term of entropy usually refers to the Shannon entropy, which quantifies the expected value of the information contained in a message (Lu et al. 2005). Entropy is typically measured in bits, nats, or bans. Shannon entropy is the average unpredictability in a random variable, which is equivalent to its information content. Shannon entropy provides an absolute limit on the best possible lossless encoding or compression of any communication, assuming that the communication may be represented as a sequence of independent and identically distributed random variables (Yuheng 1989; Lu et al. 2005). Therefore, the entropy of tunnel system evolution could be characterized by the Shannon entropy in information theory.

The energy evolution process of the dynamic instability of a tunnel system will be studied in this paper by using the dissipative structure theory. By using thermodynamic laws and information methods, an evolution model and failure criterion from a dynamic instability analysis of a tunnel system will be established.

\section{Whole Process of Energy Dissipation in Tunnel System Instability}

According to the dissipative structure theory, the tunnel system instability is an exchanging process of energy and material between the tunnel system and external environment. Therefore, the tunnel system as a whole is a non-linear open system, and the energy dissipation runs throughout the whole process of the evolution of the dynamic instability of the tunnel. In general, the tunnel deformation will undergo elastic, 
plastic and loosening deformation (the current stress is less than the initial stress). An energy dissipation analysis that concerns excavation of the surrounding rock is discussed as follows.

Rocks are in an original stress state prior to tunnel excavation. Per the theory of dissipative structure, the rock mass system is in relative equilibrium.

The first stage is the elastic deformation stage. The stress of surrounding rock is redistributed in the early stage of tunnel excavation, and in the elastic deformation stage. From the view of the dissipative structure theory, at this stage, the system is out of balance, and a macro-reversible process does not exist internally, there is uniform spatial structure (disordered structure). At this time, the surrounding rock system is in equilibrium.

The second stage is the loosening deformation stage. With the excavation process, stress on the rock mass is continuously accumulated, and results in micro-fractures. Rock mass rupture develops with increases in stress difference, thus leading to irreversible deformation. The surrounding rock is in a plastic deformation stage at this time. In terms of the dissipative structure theory, the surrounding rock has continuously exchanged the energy with the outside world, and restricted the outside world to a certain extent, but no new structure and state are created at this time. The rock system is in near equilibrium.

The third stage is the plastic deformation stage. When a partial rupture turn upappears in the surrounding rock zone, the stress concentration becomes more severe, and the deformation rate increases, so that the rupture expands. As the failure surface continues to develop, the rupture zone increases, the deformation rate rapidly increases and the rock mass loosens until collapses takes place. At this time, the surrounding rock is a loose state. In terms of the dissipative structure theory, at this stage, the surrounding rock begins to develop from a disordered to an ordered state. They absorb the energy from the outside, and at the same time, release the energy because of development of rupture, until the system cannot maintain equilibrium; Thus it is far from equilibrium. When the surrounding rock system evolves to the critical point of instability, the system is finally in a failure mode, and the system tends to be orderly because of the fluctuation and amplification. At this point, the dissipative structure of the surrounding rock system is formed.
In the deformation and instability process of the tunnel above, the surrounding rock system exchanges matter, energy and information continuously with the outside world. And the basic reason of the energy dissipation is due to thermodynamic irreversible processes, which are the essential characteristics for the energy evolution during the instability analysis of tunnel system.

\section{Energy Evolution Model of the Dynamic Instability Tunnel Systems}

\subsection{Energy Evolution Model}

According to an analysis of the energy dissipation process in terms of the instability of tunnel systems, generally, the work of external force turns into heat dissipation and the system temperature does not rise. Therefore this can be regarded as an isothermal deformation process, and the temperature $T$ is assumed to be constant. According to the laws of the thermodynamics (Yang 1996; Cai 1998), the work increment of the external force is equal to the sum of the free energy increment and dissipation increment (Eq. 1) in the isothermal deformation process. The free energy increment $\mathrm{d} F$ on the right side in the Eq. (1) represents a recoverable change, and the dissipation increment $\mathrm{d} D_{I}$ represents an unrecoverable change

$\mathrm{d} A=\mathrm{d} F+\mathrm{d} D_{I}$

where $\mathrm{d} A$ is the incremental work caused by stress, $\mathrm{d} F$ is the free energy increment that it is the part of internal energy reduced into the external work in a thermodynamic process of a system, and $\mathrm{d} D_{I}$ is the dissipation increment.

Combined the energy of any point in the tunnel system at a time:

$E=\sigma \dot{U}=\sigma_{\mathrm{r}} \dot{U}_{\mathrm{r}}+\sigma_{\theta} \dot{U}_{\theta}$ or $E=\sigma \dot{U}=\sigma_{x} \dot{U}_{x}+\sigma_{z} \dot{U}_{z}$

where $\sigma$ is the total stress of the energy point; $\dot{U}$ is the total displacement rate; $\sigma_{\mathrm{r}}$ and $\sigma_{\theta}$ are the radial and circumferential stress respectively; $\dot{U}_{\mathrm{r}}$ and $\dot{U}_{\theta}$ are the radial and circumferential displacement rates respectively; $\sigma_{x}$ and $\sigma_{z}$ are the horizontal and vertical stresses respectively; $\dot{U}_{x}$ and $\dot{U}_{z}$ are the horizontal and vertical displacement rate respectively. It is to 
emphasize that any point energy of the tunnel system could be characterized by the displacement and stress at a time. And it is not for specific tunnel shape and depth. In this paper, $\sigma_{r}, \sigma_{\theta}, \dot{U}_{\mathrm{r}}$ and $\dot{U}_{\theta}$ are as examples to derive formulas and explain energy evolution. Hence one may have:

$\mathrm{d} A=\sigma \dot{U} \mathrm{~d} t \mathrm{~d} r \mathrm{~d} \theta=\left(\sigma_{\mathrm{r}} \dot{U}_{\mathrm{r}}+\sigma_{\theta} \dot{U}_{\theta}\right) \mathrm{d} t \mathrm{~d} r \mathrm{~d} \theta$

$\mathrm{d} F=\mathrm{d} E-T \mathrm{~d} S$

where

$\mathrm{d} E=\left(E_{\mathrm{D}}-\Delta E_{\mathrm{D}}+\Delta E\right) \mathrm{d} t \mathrm{~d} r \mathrm{~d} \theta$

where $E_{\mathrm{D}}$ is the input energy when tunneling, which can be considered to be the power of the excavation equipment; $\Delta E_{\mathrm{D}}$ is the dissipated energy of the rock mass in the tunneling process, $\Delta E_{\mathrm{D}}=\rho_{\mathrm{S}} V_{\mathrm{S}} g h_{\mathrm{S}}, \rho_{\mathrm{S}}, V_{\mathrm{S}}$ and $h_{\mathrm{S}}$ are the density, volume and height of the ground loss respectively; $\Delta E$ is the energy exchanged with the external environment of the tunnel system during the evolution process (such as disturbance by construction machinery, ground loss, surrounding rock support, so and on), and input of this energy is positive, output of this energy is negative, which is also calculated by corresponding methods.

According to thermodynamics, entropy $S$ refers to the degree of disorder of the system state. And it is expressed by the parameters of the Boltzmann constant $k\left(k=1.38 \times 10^{-23} \mathrm{~J} / \mathrm{K}\right)$ and thermodynamic probability $p$ as (Huang and $\mathrm{Hu}$ 2004; Gong 2005):

$S=-k \ln p$

Supposing that the system may be in different $m$ states, $p_{i}$ is the probability of the system in the state, from (6):

$S=-k \sum_{i=1}^{m} p_{i} \ln p_{i}$

Information and thermodynamic entropies are similar. If one information source is composed of $m$ events, and probability of each event is $p_{1}, p_{2}, \ldots \ldots, p_{m}$ respectively, then the average information amount contained in each event of this information source is:

$H=-\sum_{i=1}^{m} p_{i} \lg p_{i}$

In information theory, the statistical average is created by probability of information. $H$ is defined as the information entropy, known as the Shannon entropy (Gong 2005), which is used to describe the state of the uncertainty of system.

In Eq. 8, the number "2" is commonly used as the base of logarithm, and the unit of the amount of information is called abit (Wang 2005; Wang et al. 2006). By placing Eq. 8 is put into Eq. 7. Tthen the relationship between information entropy $H$ and thermodynamic entropy $S$ is given as:

$S=H k \ln 2$

The ratio between the thermodynamic entropy and information entropy is a constant in the same system.

According to the definition of Shannon entropy, the Kolmgorov entropy ( $K_{1}$ entropy) (Shen 2001) is introduced to accurately portray the degree of the disorder of a dynamical system. Based on the definition of the $q$ order Renyi entropy ( $K_{2}$ entropy) (Shen 2001), generally, $K_{2}$ is a good estimate of $K_{1}$

$K_{2}=-\lim _{\tau \rightarrow 0} \lim _{l \rightarrow 0} \lim _{m \rightarrow \infty} \frac{1}{m \tau} \sum_{i 0 \cdots i m} p_{i 0 \cdots i m}^{2}$

A specific calculation of the entropy is provided in the literature (Wang 2005; Wang et al. 2006).

According to the definition of the Kolmogorov entropy: $m \tau K_{2}$ is the total change in the information entropy. By Combining Eqs. 8, 9 and 10, the following Eq. 11 is obtained

$S=m \tau K_{2} k \ln 2$

Equations 9 and 11 relates to the thermodynamic entropy $S$, Shannon entropy $H$, Kolmgorov entropy (referred to as $K_{1}$ entropy), q-order Renyi entropy (referred to as $K_{2}$ entropy) in discussion. Where the thermodynamic entropy Sis necessary for Eq. 9 to describe energy evolution of tunnel system. But the thermodynamic entropy Sis difficult to measure and calculate. And the thermodynamic entropy $S$ could be just right expressed by Shannon entropy $H$. In general, Shannon entropy $H$ is calculate and represented by $K_{1}$ entropy. However, the calculation of $K_{1}$ entropy is very complex. And $K_{2}$ entropy is a very good estimate of $K_{1}$ entropy and is easily to calculate. Then $K_{1}$ entropy is generally represented by $K_{2}$ entropy. Therefore, we chose $K_{2}$ entropy to calculate entropy $S$ and describe energy evolution of tunnel system finally.

By inserting Eqs. 5 and 11 into Eq. 4, one is able to obtain: 


$$
\begin{aligned}
\mathrm{d} F & =\mathrm{d} E-T \mathrm{~d} S \\
& =\left(E_{\mathrm{D}}-\Delta E_{\mathrm{D}}+\Delta E-T m \tau K_{2} k \ln 2\right) \mathrm{d} t \mathrm{~d} r \mathrm{~d} \theta
\end{aligned}
$$

Then

$$
\begin{aligned}
\mathrm{d} D_{I}= & \mathrm{d} A-\mathrm{d} F \\
= & {\left[\left(\sigma_{\mathrm{r}} \dot{U}_{\mathrm{r}}+\sigma_{\theta} \dot{U}_{\theta}\right)-\left(E_{\mathrm{D}}-\Delta E_{\mathrm{D}}+\Delta E\right.\right.} \\
& \left.\left.-T m \tau K_{2} k \ln 2\right)\right] \mathrm{d} t \mathrm{~d} r \mathrm{~d} \theta
\end{aligned}
$$

According to the assumption of local equilibrium per the dissipative structure theory (Cai 1998), the tunnel system is entirely non- equilibrium from the dynamic evolution of the instability of the tunnel system. The change of the thermodynamic state parameters could be neglected in a micro-region when the micro-region is adequately small. Then the micro-region could be considered to be in the thermodynamic equilibrium state, which is also referred to as local equilibrium. Thus the classical thermodynamics could be used for this micro-region. For asymmetrical tunnel, the radius $r_{\mathrm{j}}$ of the microregion (annular area) is divided into different ranges of the corresponding entropy $S$. The dissipation increment of this micro-region $\mathrm{d} D_{I}$ could be seen as nearly identical. So the total dissipation increment of the micro-region is:

$D_{I k}=\int_{0}^{2 \pi} \int_{r_{j}}^{r_{j+1}} d D_{I j}$

However, the equilibrium state parameters is not the same for any two different micro-regions, and the $r_{j}$ which corresponds to the dissipation increment $D_{I j}$ is not continuous in the same range of entropy $S$ range. Hence a summation form is used to describe the dissipation increment $D_{I j}$ in the same range of entropy $S$

$\sum_{j=1}^{n} D_{I j}=\sum_{j=1}^{n} \int_{0}^{2 \pi} \int_{r_{j}}^{r_{j+1}} d D_{I j}$

Equation 15 gives the degree of the influence and distribution of dissipation increment $D_{I j}$ in the same range of entropy $S$ in the process of the energy dissipation of the dynamic evolution of the whole tunnel system. The ratio of integral $\sum_{k=1}^{n} D_{I j}$ and integral $\mathrm{d} D_{I}$ presents the degree of the evolution of energy dissipation in a tunnel system (Eq. 16) $\frac{\sum_{j=1}^{n} D_{I j}}{\int_{0}^{2 \pi} \int_{r_{0}}^{r} d D_{I}}=\frac{\sum_{j=1}^{n} \int_{0}^{2 \pi} \int_{r_{j}}^{r_{j+1}} d D_{I j}}{\int_{0}^{2 \pi} \int_{r_{0}}^{r} d D_{I}}$

On the basis of the degree of evolution of the energy dissipation in a tunnel system presented by the above equation, an energy dissipation model of the evolution of the dynamic instability in a tunnel, according to thermodynamic basic laws and the dissipative structure theory, is developed from the relationship between dissipation energy and external work

$E_{E}=\frac{\sum_{j=1}^{n} \int_{0}^{2 \pi} \int_{r_{j}}^{r_{j+1}} d D_{I j} / \int_{0}^{2 \pi} \int_{r_{0}}^{r} d D_{I}}{\int_{0}^{2 \pi} \int_{r_{0}}^{r} d D_{I} / \int_{0}^{2 \pi} \int_{r_{0}}^{r} d A}$

Where $n$ is the number of zone partition, $r$ is the radius of the tunneling effect (generally less than the buried depth or calculated range $6 r_{0}$ of stress). $r_{0}$ is the radius of tunneling, $r_{j}$ is the radius of different zones and the value is determined by the division of different value ranges of entropy $K_{2}$. From Eq. 11, when $K_{2}=0$, this is in regular motion. When $K_{2} \rightarrow \infty$, this is in random motion. When $K_{2}$ is a constant greater than 0 , this is deterministic chaotic motion. A greater the $K_{2}$ of the system means greater the relative rate of information loss. This range can also be subdivided according to the system description.

Equation (17) describes the whole process of the energy evolution of the dynamic instability of a tunnel system, which is the energy evolution model of the dynamic instability of a tunnel system established in this paper.

\subsection{Degree of Stability Evolution}

According to the mathematical statistics of asymptotic distribution for dynamics analysis by Brock Dechert and Scheinkman (Lu et al. 2005), when we analyze the nonlinear time series and identifying the characteristics of chaos, the amount of data $<100$ is difficultly reflect the data evolution, the amount of data more than 3,000 is more fully reflect the data evolution, and the amount of data more than 3,000 and $<100$ is interposed between the amount of data 100 and 3,000 for reflecting evolution. Thus, by analyzing the structure and data types of the dynamical evolution model (Eq. 17) and by combining displacement and time series length in monitoring, an energy criterion in 
Table 1 The energy criteria of tunnel stability evolution degree under the conditions of large data quantity

\begin{tabular}{llll}
\hline$E_{E}$ & $\begin{array}{l}\text { Energy evolution } \\
\text { degree }\end{array}$ & Evolution types & Stability degree \\
\hline$>1$ & Low & $\begin{array}{c}\text { Low dissipative } \\
\text { type } \\
\text { General } \\
\text { dissipative type }\end{array}$ & Stable entirely \\
$=1$ & Medium & $\begin{array}{c}\text { Uncertain } \\
\text { type dissipative }\end{array}$ & $\begin{array}{c}\text { Tend to be unstable } \\
\text { possibly }\end{array}$ \\
\hline 1 & High & . & \\
\hline
\end{tabular}

the evolution process of stability for a tunnel system with the evolution of time is proposed.

1. With a large amount of data (sample size $>3,000$ ), the system is stable and the evolution shows low dissipation based on the calculation of the energy evolution model (): when; when, the stability of the system is uncertain, and the evolution generally shows dissipation; and when, the system tends to be unstable, and evolution is highly dissipative. Therefore, can be regarded as the critical point, which divides the degree of tunnel stability into three levels: low, medium and high. This is the energy criterion in the evolution process of the stability of a tunnel system by using large amounts of data (Table 1).

In addition to the criteria in Table 1, the following situation could exist. The tunnel system has not experienced any disturbances, and is in a very stable state all the time is extremely small, and the degree of energy evolution of the system cannot be distinguished by using Table 1 . This is the ideal state, in that no disturbance exists in the actual project. Therefore, this article will not discuss this case.

2. The energy criterion of the degree of evolution of tunnel stability with a small sample size $(<3,000)$ is the same as that for a large sample size. However, when the sample size is very small, the ideal state with a large sample size will also appear, that is, is very small, and all three cases are likely to occur, so then the degree of the evolution of the system stability cannot be discriminated by using Table 1 .

Therefore, according to Eq. 13, dissipation increment $D_{I}$ of tunnel system energy evolution is calculated by monitoring data. And $E_{E}$ is gotten by Eq. 17. Then, the evolution degree of tunnel system stability is obtained by $E_{E}$ comparison with Table 1 after analyzing the amount of monitoring data.

\section{Energy Criterion of Tunnel System Instability}

On the basis of the energy evolution model of the tunnel systems instability, as well as the concept of correlation dimension, the integration of the energy dissipation for the regional correlation dimension for the entire system at a certain time $t$ can be expressed as:

$C_{m 1,2,3}^{E}=\frac{\sum_{j=1}^{m}\left(\pi r_{j+1}^{2}-\pi r_{j}^{2}\right)}{\pi r^{2}-\pi r_{0}^{2}}$

where $\mathrm{k}$ is the arbitrary number of zones in a tunnel system, and 1,2, 3 respectively represent the regular motion state, random motion state and chaotic oscillatory state.

Obviously, the integration of the system energy evolution for the regional correlation dimension of the system at the time $t C_{m 3}^{E}$ (Eq. 18) is consistent with $E_{E}$ (Eq. 17) at the moment $t$, based on the surrounding rock partition of entropy $S\left(K_{2}\right)$. That $r$ is the radius of tunneling effect (it is general less than buried depth or calculation range $6 \mathrm{r}_{0}$ of stress). $r_{0}$ is the radius of tunneling, $r_{j}$ is the radius of different zone and be valued by division of different entropy $K_{2}$ value range. At the same time, in space, it can fully describe the energy evolution state of the system at time $t$. Thus, according to the basic characteristics of the negative entropy and dimensionality reduction of dissipative structures, Eq. (18) can be used as a stable criterion of the energy evolution at time $t$.

The energy criterion of the dynamic failure of a tunnel system due to instability is composed of: (1) the negative entropy and dimension reduction of some of the zones, (2) a series of variations in the rate of the correlation dimension for zone integrals: $\frac{\partial C_{m 1}^{E}}{\partial t}, \frac{\partial C_{m 2}^{E}}{\partial t}$ or, and (3) a series of variations in the rate of the correlation dimension for the zone integral of the dissipation energy in the plastic zones.

The physical meaning of the above criterion is given as: (1) the correlation dimension or entropy $K_{2}$ in a region is too large, thus indicating that this region has a higher degree of energy dissipation (meaning instability), and the tunnel will be locally instable, (2) a series of variations in the regional plotting of the 
correlation dimension appear to have reduction in entropy or dimensionality for three kinds of conditions, which demonstrate that the entire tunnel is in an unstable state, and a state with frequent changes, (3) unstable changes in the entire plastic zone within its associated integrated changing rate series or the region appears to have dissipative structure trends, or even formed a dissipative structure, thus indicating that the tunnel system is instable and there is impending destruction, and urgent measures and actions are needed to control the stability of the tunnel. As state above, the degree of dissipation in (1)-(3) is constantly increasing, so the criterion can also determine the severity of the instability of the tunnel system. Therefore, this is the energy criterion for the instability and failure of the tunnel.

According to the above analysis, the criterion is based on monitoring data. So it is required that the monitoring distribution can reflect the overall changes in the lining and surrounding rock, and a sufficient sample size (greater than 3,000) is required.

Therefore, we could calculate entropy $K_{2}$ of tunnel system by monitoring data. And the radius of different zone in tunnel system is valued by division of different entropy $K_{2}$ value range. Then, according to Eq. 18 and the energy criterion of tunnel system instability failure, correlation dimension, entropy $K_{2}$ and regional correlation dimension integration of different zone is gotten to discriminate the status of tunnel system instability.

\section{Engineering Application}

The energy evolution model and critical failure criterion of tunnel system is studied based upon the project of an overlapped shield tunnel groups, with an automatic passenger transportation system located at Line 1 of the metro in Zhujiang New Town, GuangZhou, China.

This project involves the utilization of the passenger automatic transportation system of Guangzhou Pearl River NewTown, also known as a centralized transportation system. It was constructed to pass from the Concourse station to the Tianhenanyilu station in Hongcheng Park. The shield was to pass through Tiyuxi-Tiyuzhongxin of Guangzhou metro Line 1, in Tianhenanyilu. The whole lot of overlapped tunnels

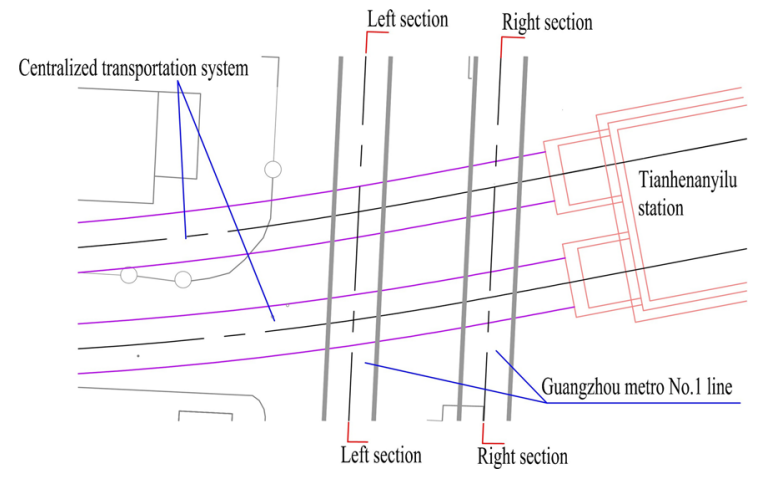

Fig. 1 Plane relative position of shield zone and Line 1 (JJJZ002 2007)

groups, consisted of two twin tunnels overlapping another set of twin tunnels. Figure 1 shows the plane view of the relative position of centralized transportation system and Guangzhou metroLine 1. It should be noted that this particular project was constructed to pass through the bottom of the municipal main roads. Its peripheral environment had to be considered because of its sensitivity to the high-density buildings and heavy traffic flow at the project location (JJJZ002 2007).

The foundation pit of Tianhenanyilu station was constructed before tunnel crossing. The nearest distance between the foundation pit and Line 1 tunnel is $2.36 \mathrm{~m}$. The centralized transportation system (shield tunnels) passed under Line 1 (existing tunnels) in September 2007 and June 2008, twice. The construction of Line 1 had been heavily disturbed, three times. In order to ensure the safety during project, the soils in the overlapped location were reinforced by grouting methods before crossing occurred. The buried depth of shield tunnels is about $17.54-17.89 \mathrm{~m}$. The distance between the left tunnel and right tunnel is $13 \mathrm{~m}$, and the distance between shield tunnels and mined tunnels is $2.275 \mathrm{~m}$. An earth pressure, balance shield constructed the centralized transportation system. Its outer diameter measured $6.28 \mathrm{~m}$ and its inner diameter measured $6 \mathrm{~m}$. The width of the tunnel segment measured $1.5 \mathrm{~m}$ per ring. The tunnel segment connected by means of bending bolts in longitudinal and lateral directions (JJJZ002 2007) (Table 2).

The plane relative position of shield zone and metro No. 1 line is shown in Fig. 1, and the longitudinal relative position is shown in Fig. 2. The soil profile 
Table 2 Physical-mechanical parameters of each stratum (JJJZ002 2007)

\begin{tabular}{|c|c|c|c|c|c|c|c|c|c|}
\hline Stratified & Name & $\begin{array}{l}\rho(\mathrm{g} / \\
\left.\mathrm{cm}^{3}\right)\end{array}$ & $\mathrm{c}(\mathrm{kPa})$ & $\varphi\left({ }^{\circ}\right)$ & $\begin{array}{l}\mathrm{E}_{\mathrm{O}} \\
(\mathrm{MPa})\end{array}$ & $\mu$ & $\mathrm{K}(\mathrm{m} / \mathrm{d})$ & $\mathrm{k}_{0}$ & $\mathrm{M}(\mathrm{m})$ \\
\hline$<1>$ & Artificial filled soil & 1.8 & 10 & 10 & 18 & 0.2 & 0.5 & 0.67 & $3.2-3.4$ \\
\hline$<3-1>$ & Fine sand & 2 & 0 & 27 & 4.5 & 0.29 & 2.0 & 0.4 & $0.7-0.8$ \\
\hline$<3-2>$ & Medium-coarse sand & 2 & 0 & 30 & 8.0 & 0.26 & 3.0 & 0.36 & $0.4-1.8$ \\
\hline$<4-1>$ & Cohesive soil & 2 & 24.4 & 12.1 & 15 & 0.32 & 0.01 & 0.47 & $3.3-6.3$ \\
\hline$<5-1>$ & Plastic residual soil & 1.93 & 16.6 & 10.9 & 15 & 0.32 & 0.005 & 0.48 & $3.1-3.4$ \\
\hline$<5-2>$ & Firm residual soil & 2.02 & 27.2 & 14.4 & 20 & 0.3 & 0.005 & 0.43 & $2.5-3.2$ \\
\hline$<6>$ & Completely weathered red beds & 2.06 & 34.9 & 16.1 & 34 & 0.26 & 0.01 & 0.36 & $1.1-2.8$ \\
\hline$<7>$ & Strongly weathered red beds & 2.2 & 60 & 25 & 195 & 0.25 & 0.1 & 0.33 & $0.8-4.9$ \\
\hline$<8>$ & $\begin{array}{l}\text { Intermediary weathered argillaceous } \\
\text { siltstone }\end{array}$ & 2.5 & 200 & 28 & 502 & 0.24 & 0.1 & 0.25 & $0.6-4.5$ \\
\hline$<9>$ & Slightly weathered argillaceous siltstone & 2.63 & 800 & 35 & 856 & 0.24 & 0.01 & 0.22 & $2-17.5$ \\
\hline$<9>$ & Slightly weathered conglomerate & 2.67 & 1,200 & 38 & 1,988 & 0.18 & 0.01 & 0.22 & $6-11.1$ \\
\hline
\end{tabular}

* $\rho$ Natural density, $c$ cohesion, $\varphi$ the angle of internal friction, $E_{O}$, deformation modulus, $\mu$ Poisson's ratio, $K$ permeability coefficient, $k_{0}$ lateral pressure coefficient, $M$ formation thickness
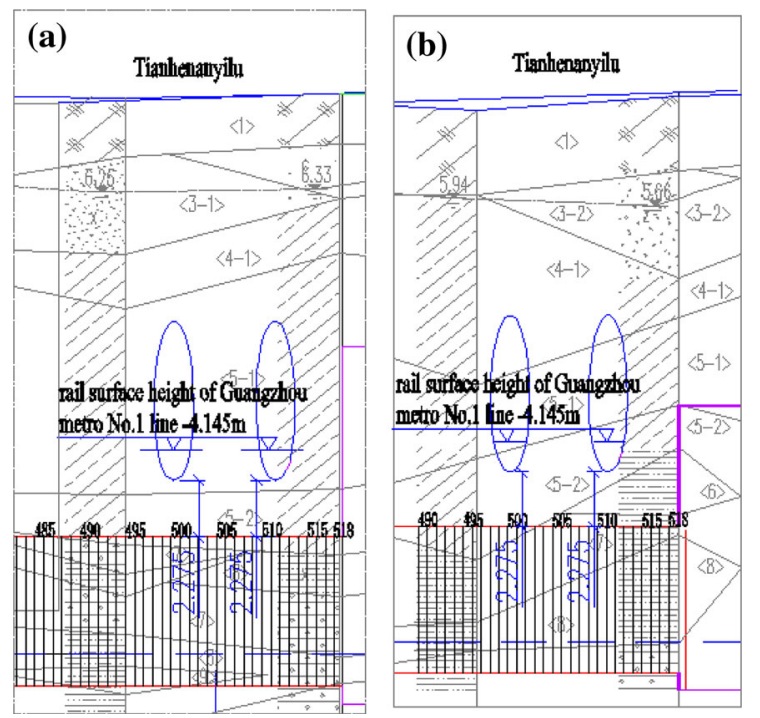

Fig. 2 Longitudinal relative position of shield zone and metro Line 1 (JJJZ002 2007)

starting from the ground surface to the shield tunnel roof is summarized in Table 3, which includes the physical-mechanical parameters of each stratum.

Moreover, from January 2007 to September 2008, the elevation displacement, lateral displacement and vertical displacement are monitored at the under crossed process of metro Line 1, and the monitoring interval was $1-4 \mathrm{~h}$, which is considered to be frequent.
Table 3 Final state entropy K2 of all monitored points. a Left section. b Right section

\begin{tabular}{llllll}
\hline Sections in & Point 1 & Point 2 & Point 3 & Point 4 & Point 5
\end{tabular} monitoring

points

\begin{tabular}{llllll}
\hline S-ADD1 & 0.0156 & $/$ & 0.0093 & 0.0065 & 0.0017 \\
S-ADD2 & 0.0042 & 0.0011 & 0.0019 & 0.0028 & 0.0013 \\
S-ADD3 & 0.0048 & $/$ & 0.0049 & 0.0009 & 0.0036 \\
S-ADD4 & 0.0022 & 0.0035 & 0.0036 & 0.0057 & 0.0014 \\
S-ADD5 & 0.0032 & 0.0007 & 0.0021 & 0.0036 & 0.0025 \\
S-ADD6 & 0.0051 & 0.0024 & 0.0055 & 0.0011 & 0.0026 \\
SA & 0.0021 & 0.0059 & 0.0017 & 0.0013 & 0.0016 \\
SB & 0.0026 & 0.0029 & 0.0032 & 0.0029 & 0.0036 \\
SC & 0.008 & 0.0038 & 0.0026 & 0.0021 & 0.0052 \\
SD & 0.0013 & 0.0012 & 0.0028 & 0.0034 & 0.0012 \\
SE & 0.0041 & 0.0017 & 0.0005 & 0.0024 & 0.0014 \\
X-ADD1 & 0.0018 & 0.0025 & 0.0006 & 0.0022 & 0.0015 \\
X-ADD2 & 0.0043 & 0.0059 & 0.0023 & 0.0015 & 0.0011 \\
X-ADD3 & 0.0013 & 0.0021 & 0.0016 & 0.0017 & 0.0026 \\
X-ADD4 & 0.0012 & 0.0011 & 0.0004 & 0.0052 & 0.0035 \\
X-ADD5 & 0.0019 & 0.0046 & 0.0026 & 0.0038 & 0.0015 \\
X-ADD6 & 0.0037 & 0.0022 & 0.0011 & 0.0029 & 0.0035 \\
X A & 0.0018 & 0.0013 & 0.0037 & 0.0016 & 0.0027 \\
X B & 0.0022 & 0.0019 & 0.0029 & 0.0031 & 0.0028 \\
X C & 0.0017 & 0.0016 & 0.007 & 0.0032 & 0.0024 \\
X D & 0.0016 & 0.0022 & 0.0014 & 0.0025 & 0.0012 \\
X E & 0.0024 & 0.0041 & 0.0028 & 0.0027 & 0.0015 \\
\hline & & & & &
\end{tabular}


Fig. 3 Monitored sections and monitored point layout ((JJJZ002 2007). a

Monitored section. b

Monitored point layout

Fig. 4 Degree of energy evolution of tunnel lining in uplink of Line $1 \mathbf{a}$, in downlink of Line $1 \mathbf{b}$

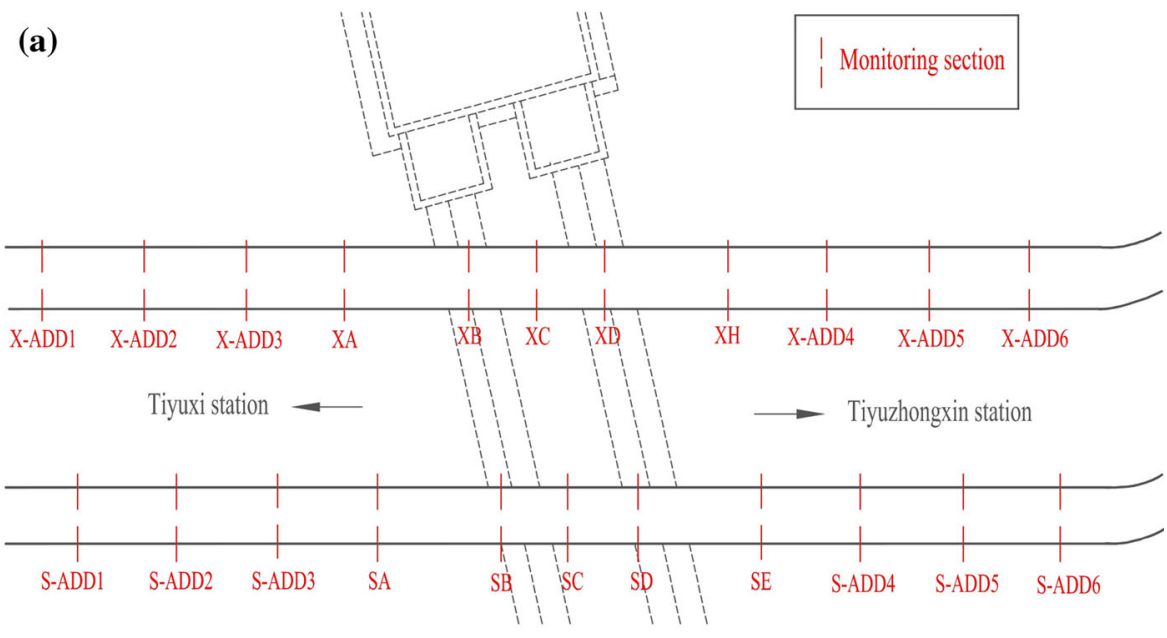

(b)
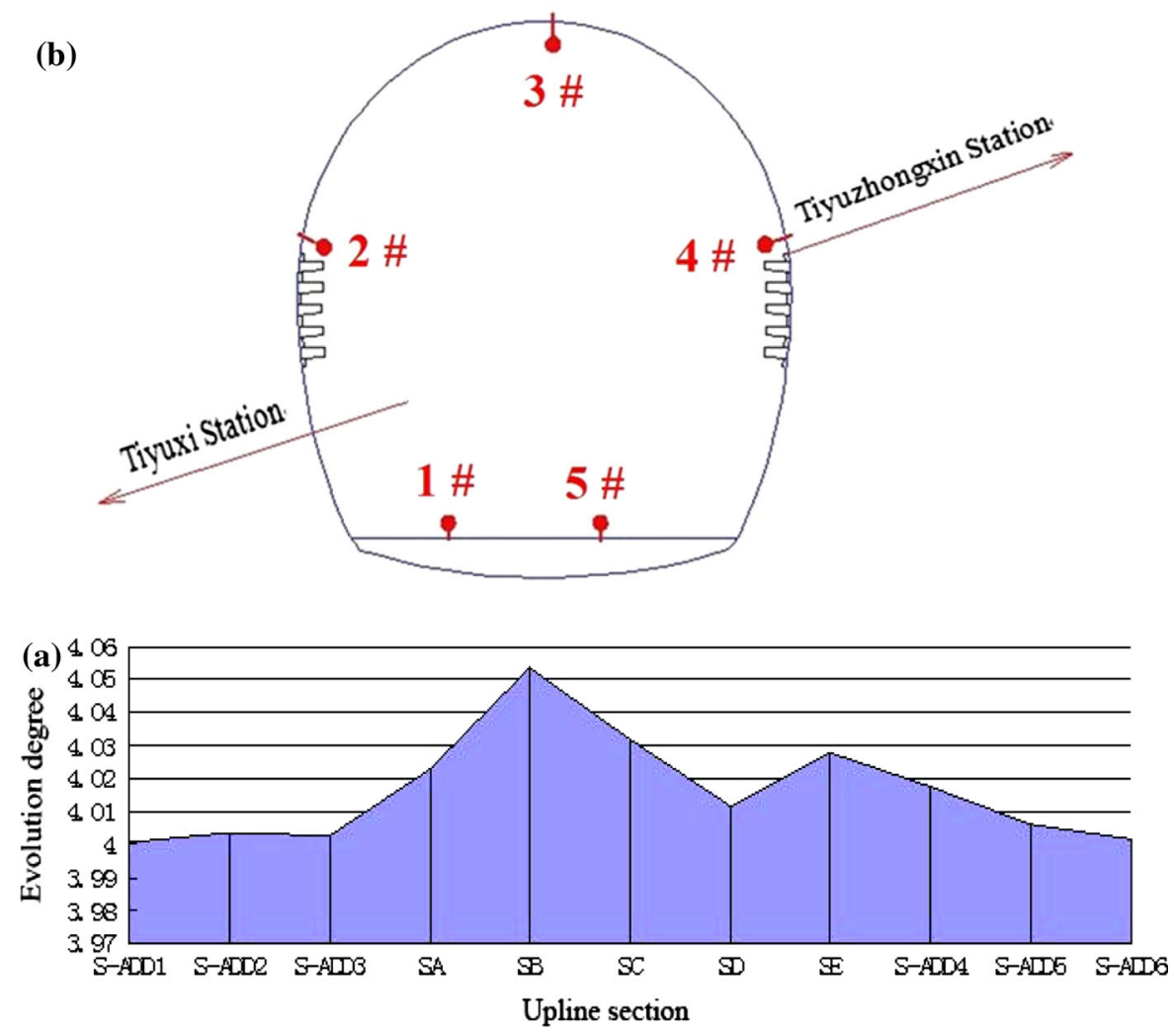

(b) 4

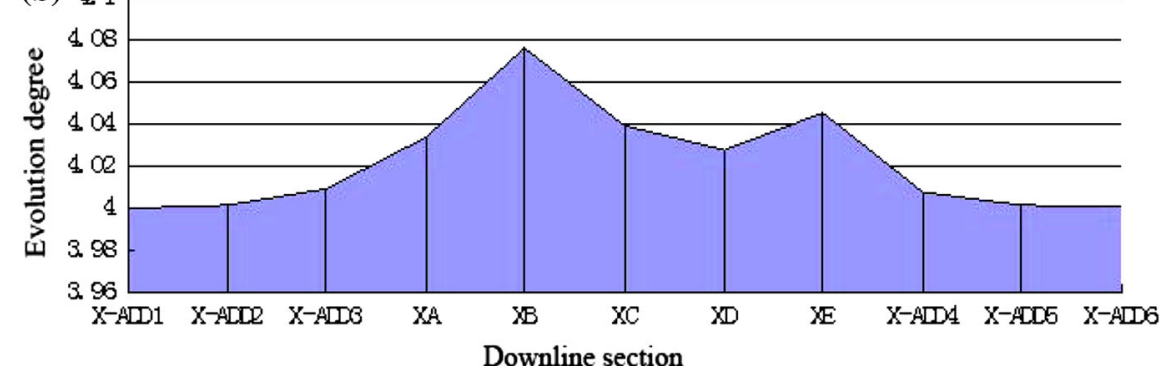


(a) X-Displacement(mm)

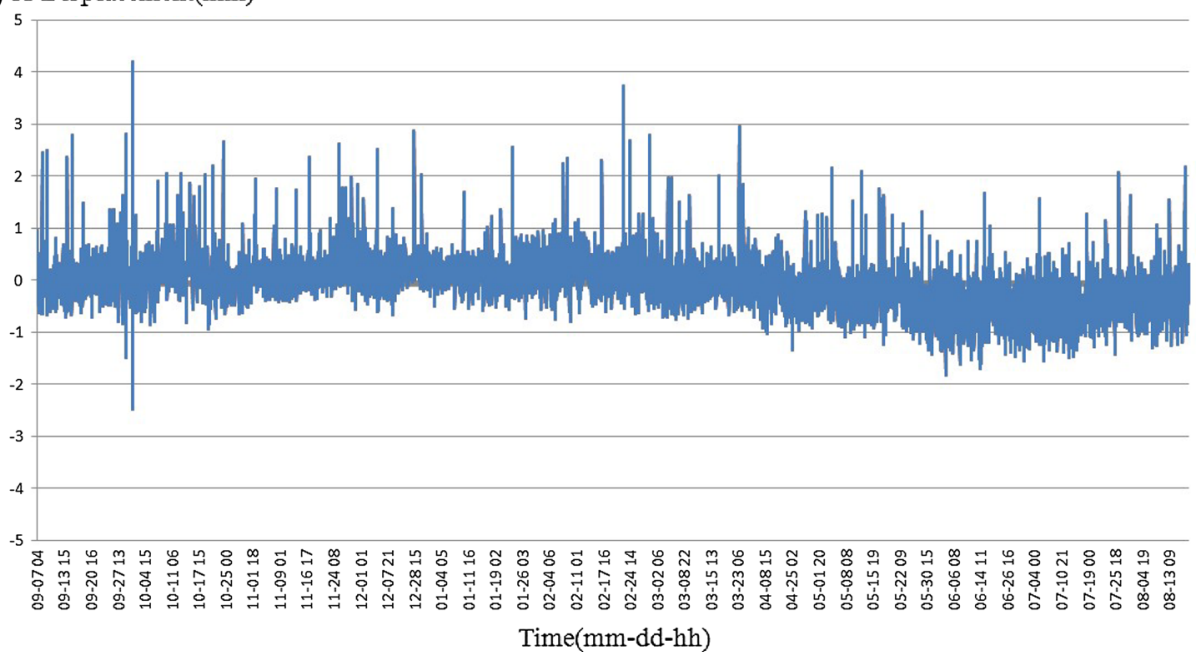

(b) Y-Displacement(mm)

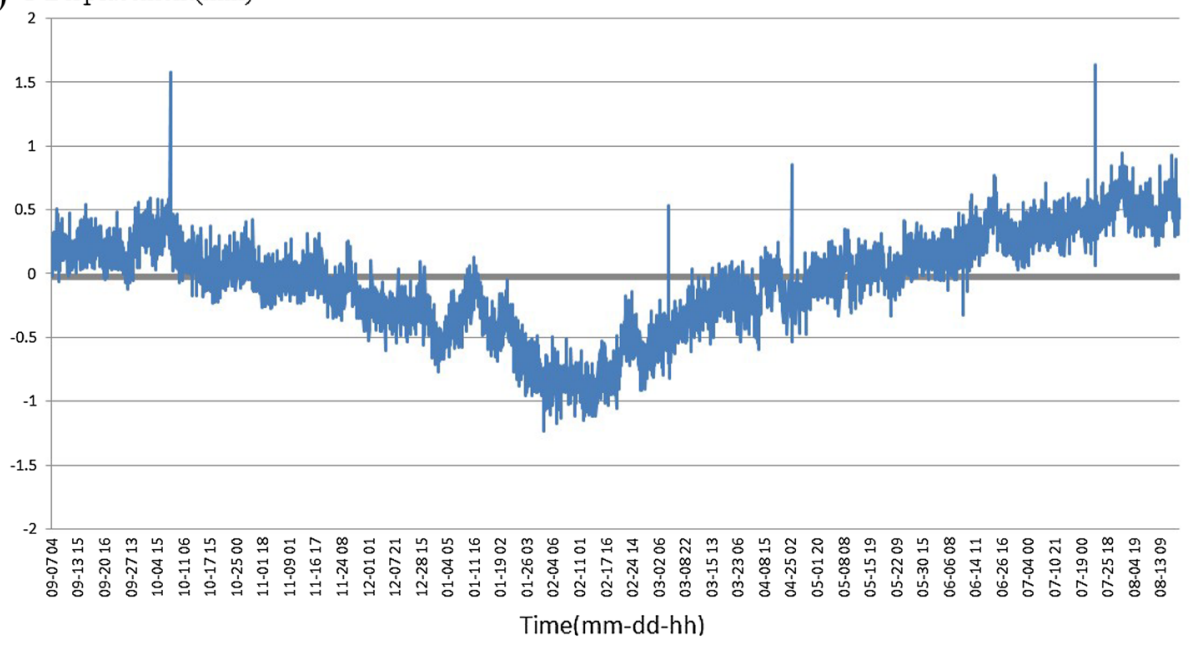

(c) Z-Displacement(mm)

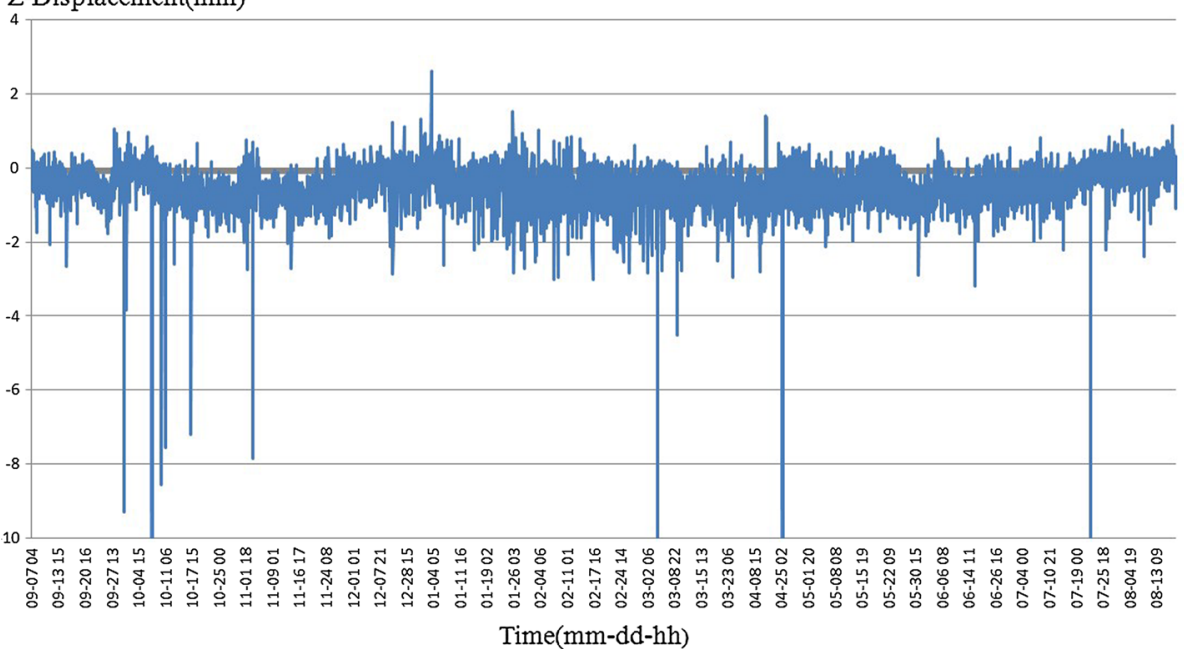

Fig. 5 The displacement curve in $x, y, z$ direction of monitored point S-ADD1-1. a The displacement curve in X direction, b the displacement curve in $\mathrm{Y}$ direction, $\mathbf{c}$ the displacement curve in $\mathrm{Z}$ direction 


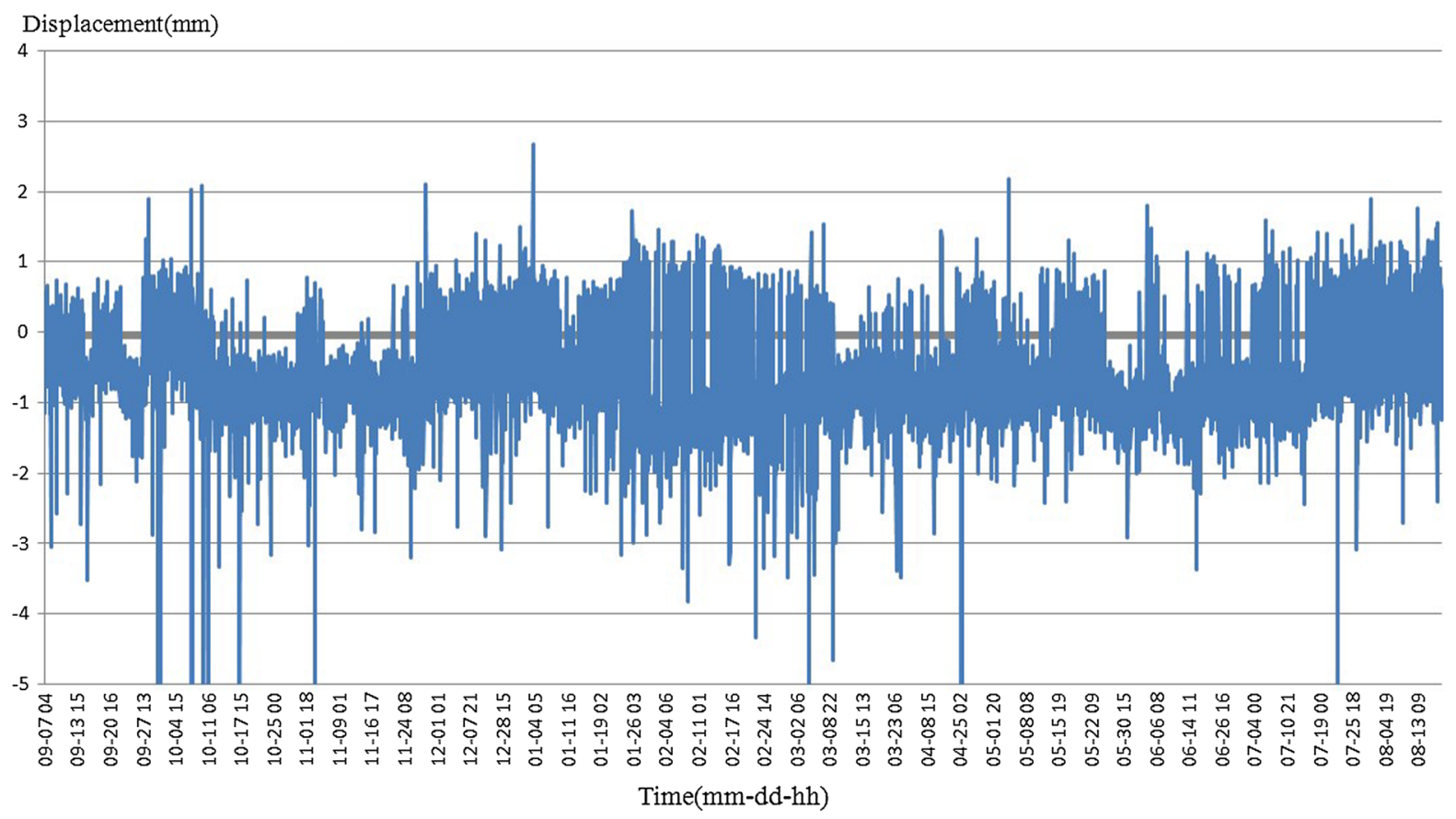

Fig. 6 The overall displacement curve of monitored point S-ADD1-1

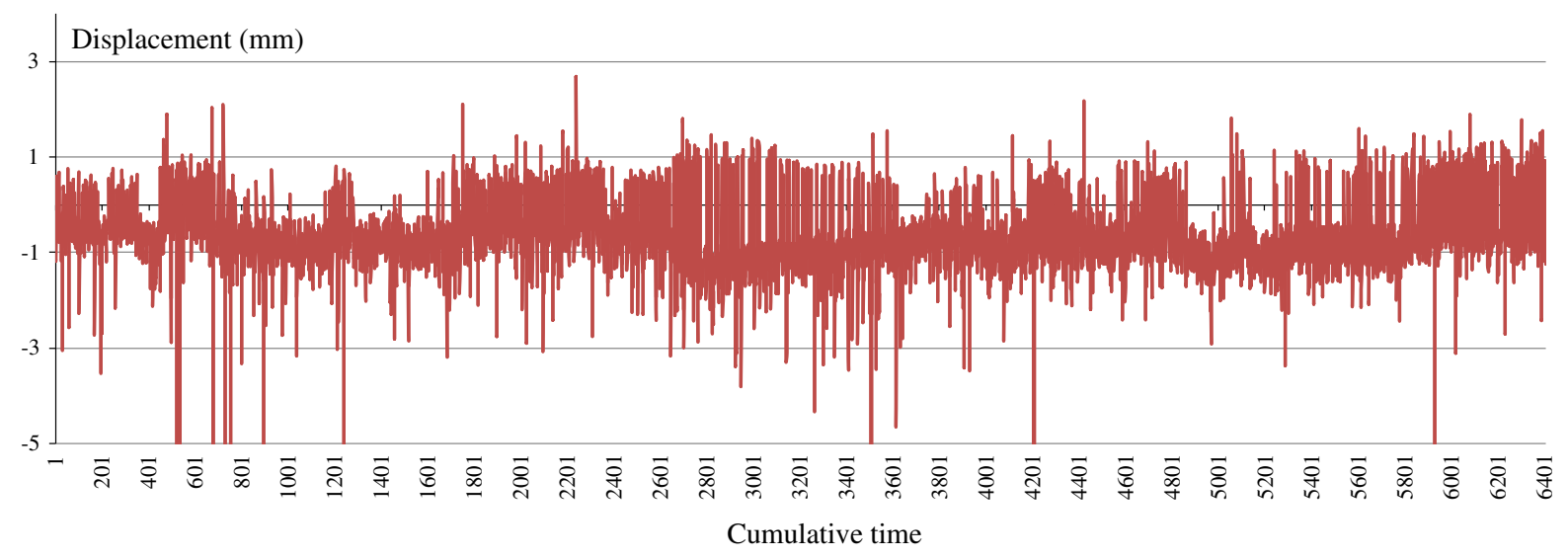

Fig. 7 The overall displacement curve of monitored point S-ADD1-1

The monitored sections and monitored point layout are shown in Fig. 3.

To verify the energy evolution model and criterion of the overlapped tunnels, first, combined with the actual conditions, the energy evolution model is applied based on the project. In accordance with the energy evolution model and criterion in tunnel system evolution, 22 sections and 110 monitored points Line 1 are analyzed. The correlation dimension and largest Lyapunov exponent are calculated to discriminate the stability. The results are compared with the actual monitored and three-dimensional finite element calculation to verify the rationality and reliability.

\subsection{Energy Evolution Analysis}

Based on a time series analysis of monitored data at 22 monitored sections on the intersection of Line 1, and in accordance with Eq. 17, the deterministic energy dissipation component of each section is analyzed to 

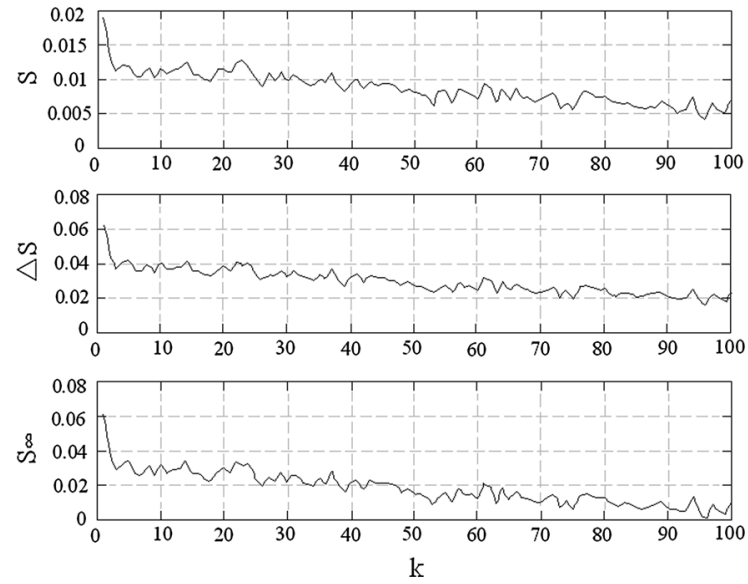

Fig. 8 Analysis curve of phase space reconstruction

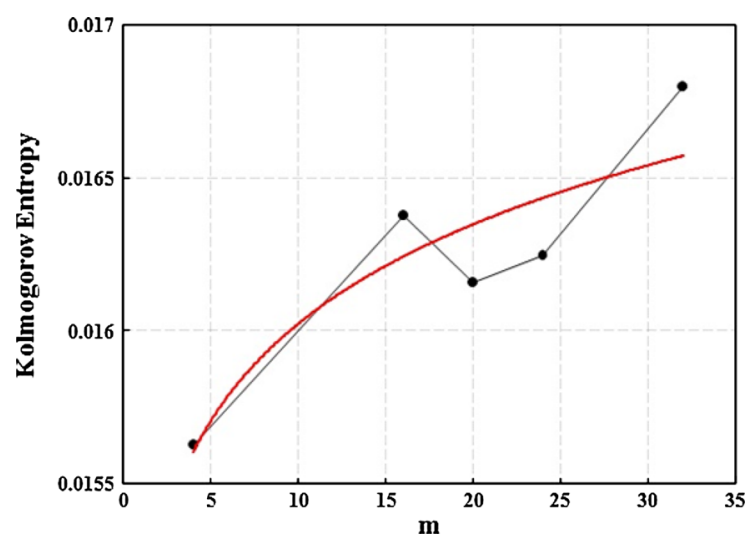

Fig. 9 Relationship between entropy $K$ and $m$

get the degree of energy evolution in the project, which is presented in Fig. 4.

As Seen from Fig. 4, in the monitoring period, the degree of energy evolution in the tunnel cross-sections is $4.0000-4.0761$. This shows that the overall energy evolution degree of the metro No. 1 line tunnel is about 4. The overall energy evolution degree 4 indicates that the tunnel system has a composition in deterministic chaotic motion, but the energy dissipation caused by this volatility component is not high. According to Table 1, it is also revealed that the evolution degree of the whole system is low and stable. The degree of the evolution of stability is nearly symmetric of lowintermediate and high-springlines along the tunnel longitudinal. These indicate that the new tunnel has a certain influence to the existing tunnel, reducing the energy dissipation components of the cross section, and it develops towards a the more stable direction. These also show that pre-grouting and buffers play the significant roles.

\subsection{Stability Discretion}

The final state of the monitored point S-ADD1-1 is taken as an example to explain the calculation process.

\subsubsection{Time Series}

from September 7, 2007 to August 18, 2008 of monitored point S-ADD1-1 are used, which is a total of 347 days, or a set of data with about 6,411 observations. The daily displacements in the $x, y, z$ direction are shown in Fig. 5, and the overall displacements are obtained by $\sqrt{x^{2}+y^{2}+z^{2}}$, as presented in Fig. 6.

\subsubsection{Phase Space Reconstruction}

The overall displacement curve of monitored point S-ADD1-1 was plotted, and spline interpolation was carried out. The monitored data were equally divided by interpolation, with a total of 6,510 points. The processed nonlinear time series is shown in Fig. 7.

Based on the embedding window method of phase space reconstruction [also known as the Kugiumtzis theory (Wang 2005)], phase space reconstruction is studied using C-C method (Wang 2005), which has strong anti-noise ability and needs less data to calculate $\tau$ and $m$. C-C method is a kinds of the embedding window methods of phase space reconstruction and used usually.

From Fig. 8, the phase space can be reconstructed by adopting the overall displacement time series of delayed time $\tau=4 \tau_{\mathrm{s}}, m=4$ to study the chaotic characteristic of this system.

On the basis of phase space reconstruction, entropy K2 can be calculated by using Matlab, and its relationship with the embedding dimension $\mathrm{m}$ is shown in Fig. 9, where the broken black line represents the actual calculated values, and smooth curves indicate fitted values. From Fig. 9, the laws of entropy $\mathrm{K} 2$ are not obvious and oscillation exists, and the system is close to 0 on the positive side. By using fitting analysis, entropy $\mathrm{K} 2$ approximately equals to 0.0156 . 

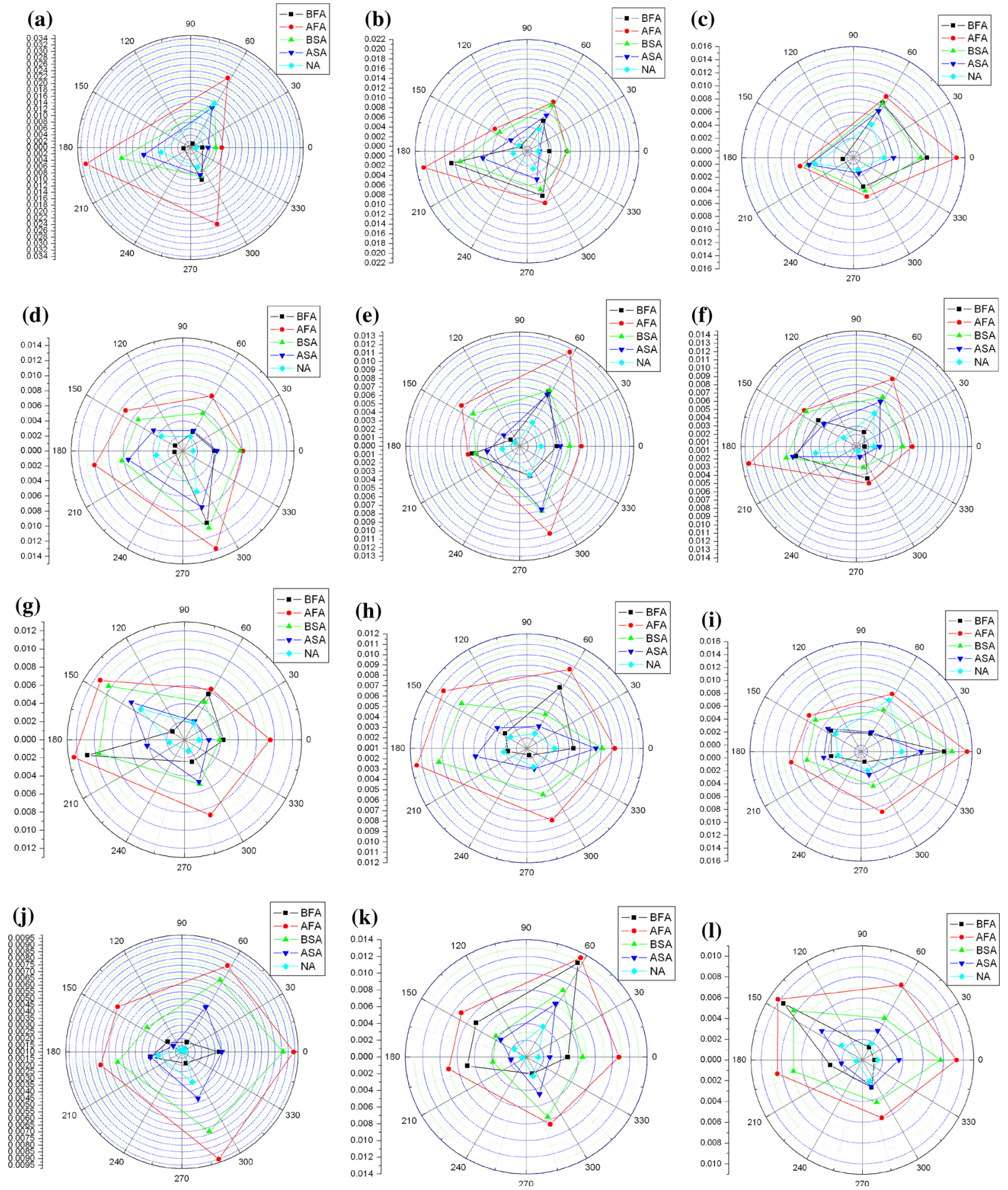

Fig. 10 Evolution of law of entropy $K_{2}$ in each monitored section of the lining. a S-ADD1 section, b S-ADD2 section, c S-ADD3 section, d S-ADD4 section, e S-ADD5 section, $\mathbf{f}$ S-ADD6 section, $\mathbf{g}$ SA section, h SB section, i SC section, $\mathbf{j}$ SD section, $\mathbf{k}$ SE section, I X-ADD1 section, $\mathbf{m}$ X-ADD2 section, $\mathbf{n}$ $\mathrm{X}$-ADD3 section, $\mathbf{o} \mathrm{X}$-ADD4 section, $\mathbf{p} \mathrm{X}$-ADD5 section, $\mathbf{q}$ $\mathrm{X}$-ADD6 section, $\mathbf{r}$ XA section, $\mathbf{s}$ XB section, $\mathbf{t}$ XC section, $\mathbf{u}$ XD section, $\mathbf{v}$ XE section 

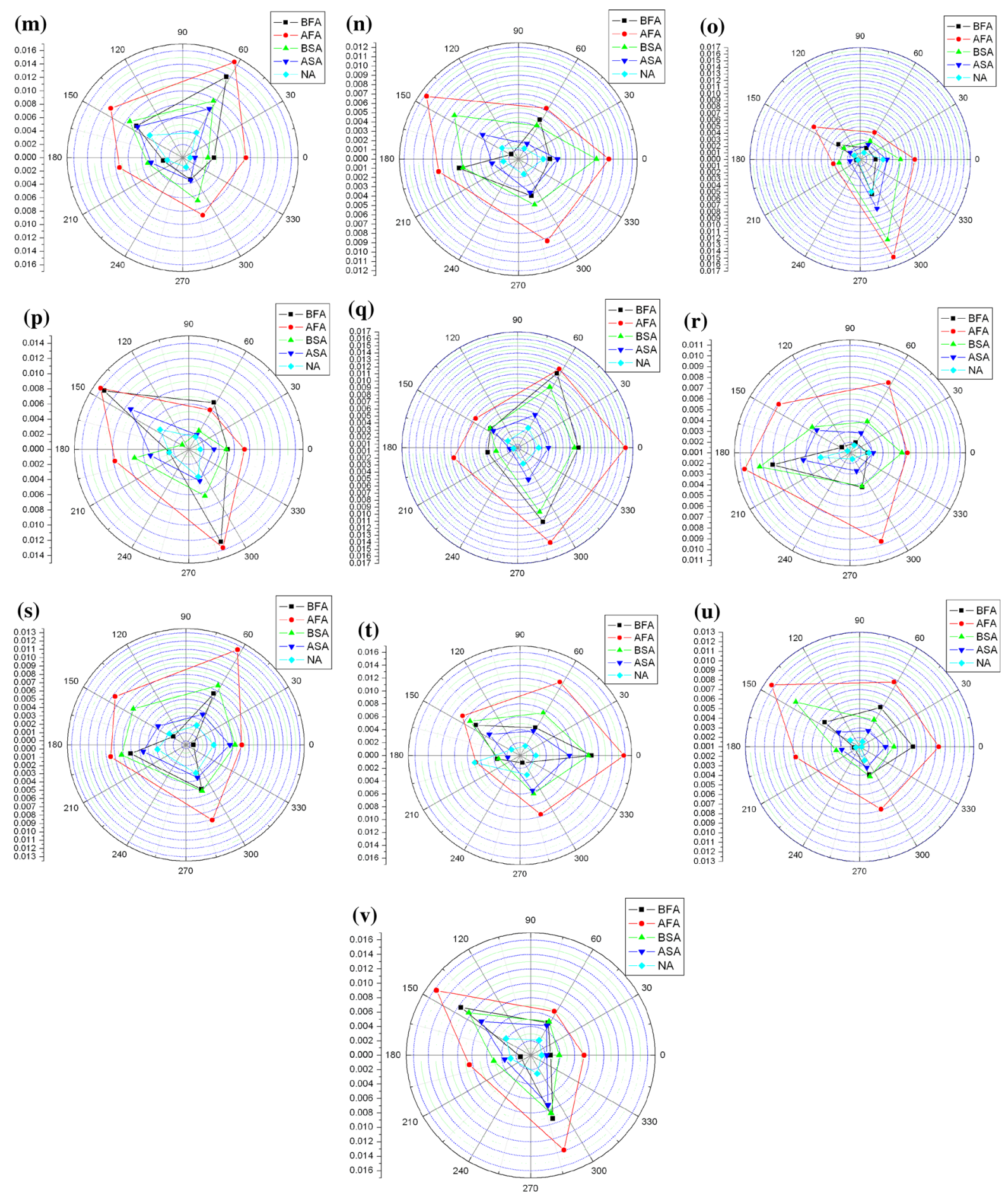

Fig. 10 continued

Meanwhile, based on phase space reconstruction, the relationship between entropy $K_{2}$ and embedding dimension $m$ is calculated. And the entropy $K_{2}$ in the final state at each monitored section with the time are confirmed by fitting analysis, shown in Table 3 and Fig. 10. According to the time interval of the 


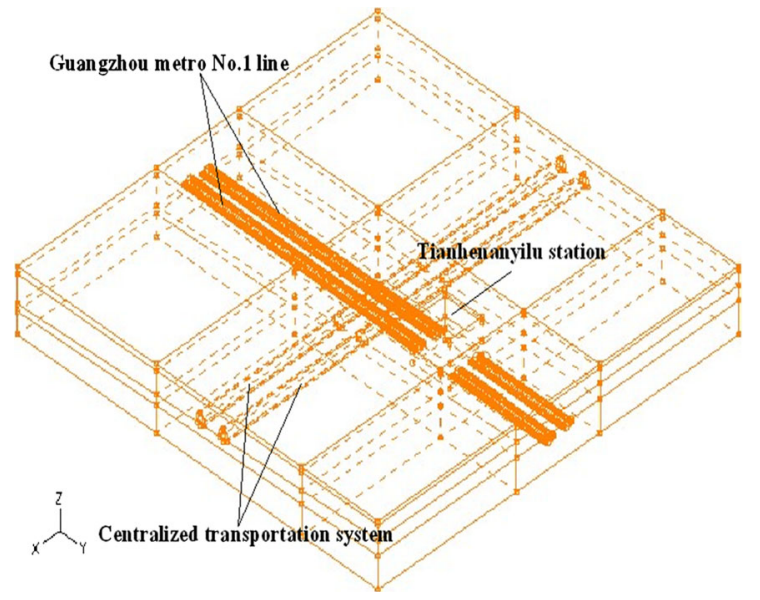

Fig. $113 D$ nonlinear finite element model of the overlapped tunnels engineering

Centralized transportation system (shield tunnels) passing through under Line 1 (existing tunnels), combined with the experience numerical calculation, the time series of each monitored point is divided into five parts, including before the first crossing (hereinafter referred to as BFA), after the first crossing (hereinafter referred to as AFA), before the second crossing (hereinafter referred to as BSA), after the second crossing (hereinafter referred to as ASA) and non-crossing period (hereinafter referred to as NA). From Fig. 10, the evolution of final state entropy $K_{2}$ of 22 monitored sections in each stage is obtained. The ordinate is the entropy $K_{2}$ in Fig. 10 . The relative position of each monitored point in Fig. 10 and the actual layout of monitored points are the same.

From Table 3, the final state entropy $K_{2}$ of all monitored points are very small, and entropy $K_{2}$ of the entire system tends to 0 on the positive side, which indicates that the whole system contains unstable trend (chaotic components), but the unstable trend is very small (the chaos degree is very low). Therefore, taking into account the data noise, train load and short time series, the tunnel is in the stage of stable equilibrium.

It can be observed in Fig. 10 that the entropy $K_{2}$ in each monitored sections of the lining region increases first from BFA to AFA and then decreases from AFA to NA. The reason is mainly due to the sudden change from the operating stage to the passing stage before the first crossing, and the displacement of each monitored point obviously changes with abrupt adjustment of the system. Then the system gradually adjusts to the status to adapt to the process of crossing. In particular, the entropy $K_{2}$ of NA is less than BFA, and the small change in the overall range, which indicates that the system recovers to the prior steady state or tend to move towards a new and more stable state. However, entropy $K_{2}$ is non-negative and increasing, which demonstrate that the new tunnel crossing enhances the chaotic component of the existing tunnel system. Combined withing the final state of the entropy $K_{2}$ from Table 3, it is confirmed that the entire system is in the stage of developing stable equilibrium during the crossing process.

In summary, based on the energy evolution criterion, by analyzing the changing process final state the entropy $K_{2}$ with time, it is clarified that the entire interchange tunnel system is in the stage of developing stable equilibrium during the crossing process.

\subsection{Three-Dimensional Finite Element Calculation}

Three-dimensional finite element modeling is very common calculation method for the tunnel stability analysis. So,a 3D nonlinear finite element model of this overlapped tunnels engineering is developed by using parasolid geometric modeling technology in ADINA (shown in Fig. 11), in order to compare with this energy evolution analysis and verify the reasonableness of this energy evolution analysis. Considering the limitation of computational-scale and convergence condition, some key factors such as the tunnel undercrossing process, surrounding rocks, segments and links, grouting layer on the back wall, shield machine pushing, friction between shield and surrounding rocks, have been simulated in detail, shown in Fig. 12. Based on the results of the simulation, the overall stability of the overlapped tunnels engineering has been evaluated comprehensively, shown in Fig. 13.

\subsection{Comparative Analysis}

The analysis above was compared with actual monitored data as listed in Table 4.

Table 4 shows that the results of the energy evolution model are consistent with the monitored results, which support that the centralized transportation system 

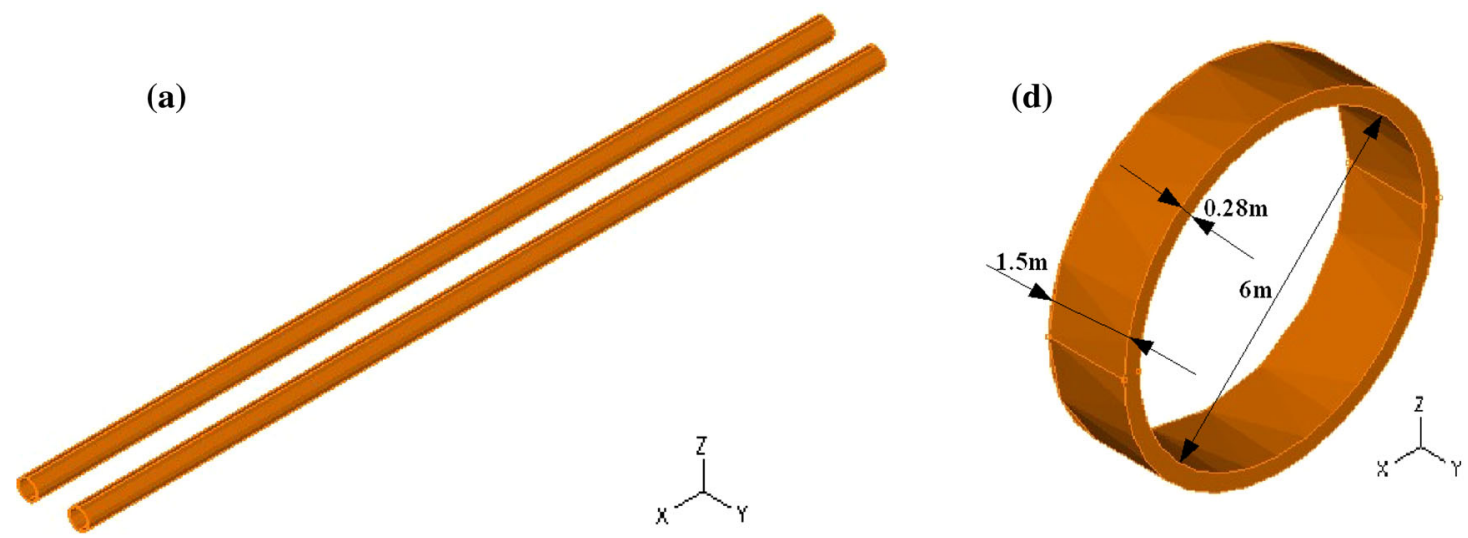

(e)
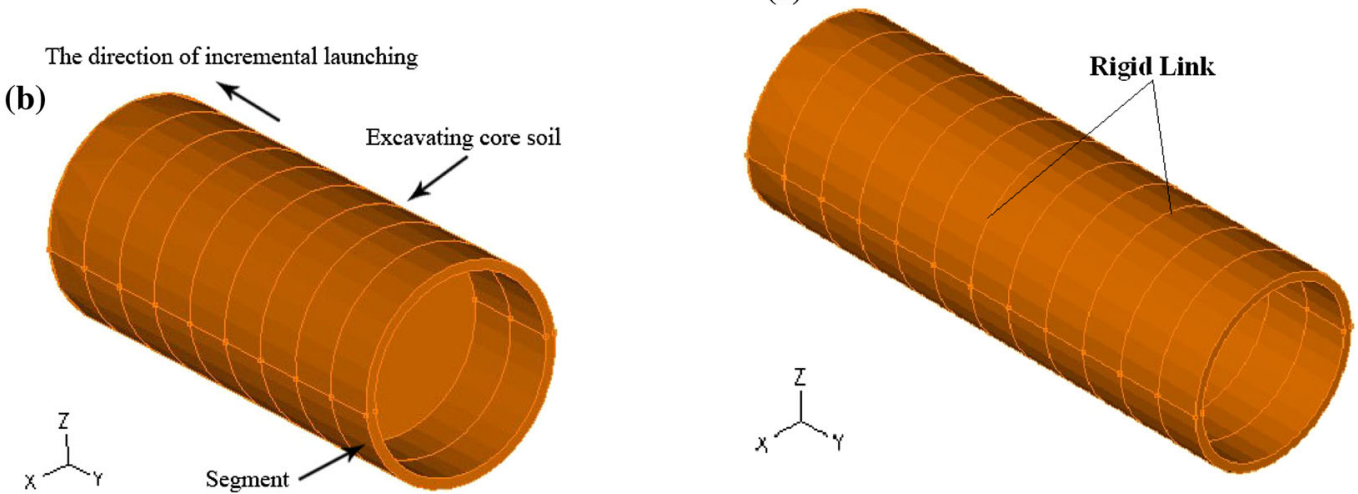

(c)
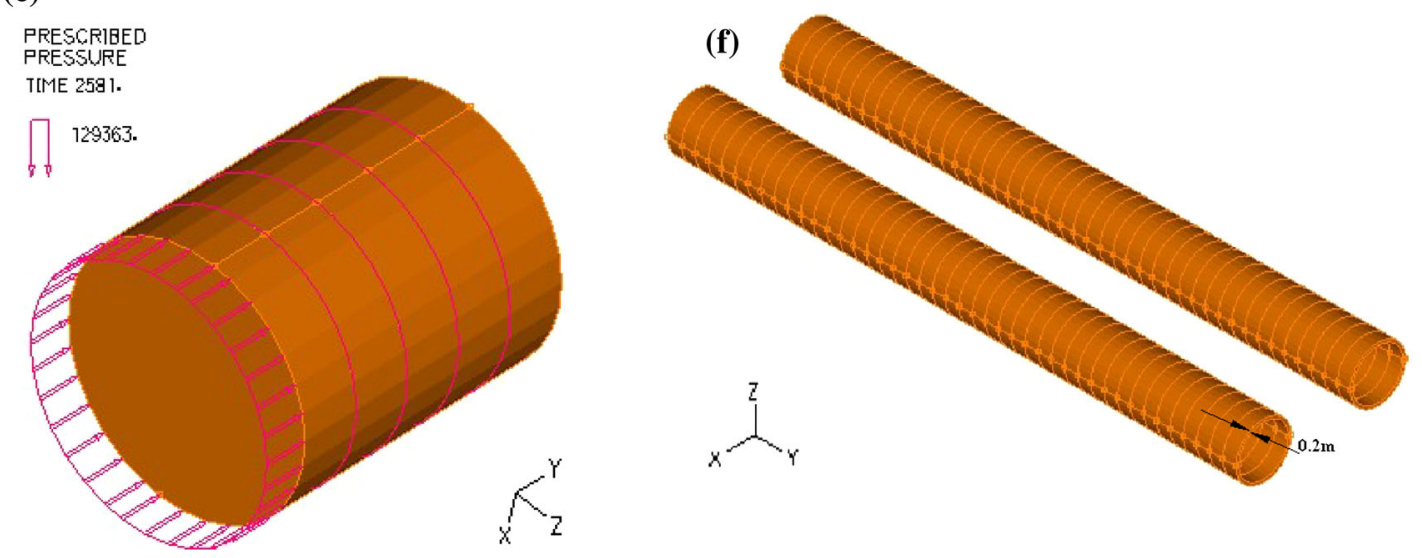

Fig. 12 The details simulation of the overlapped tunnels engineering. a Simulation of metro No.1 line mined tunnels, b shield tunneling simulation, $\mathbf{c}$ the simulation force on stratum in

crossing has little influence to Line 1, and Line 1 has always been in a stable state all along. In the meantime, the reasonability and reliability of the model and the failure criteria established in this paper are verified. This

shield tunneling process, d shield segment simulation, e simulation of connection between segments, $\mathbf{f}$ simulation of backwall grouting layerFig. 12

research has a certain reference value for the calculation and analysis of tunnel stability.

Now we only collect the information and data of this project. Therefore, the only one application in this 
(a) The distance between ground point and cross point( $\mathrm{m})$

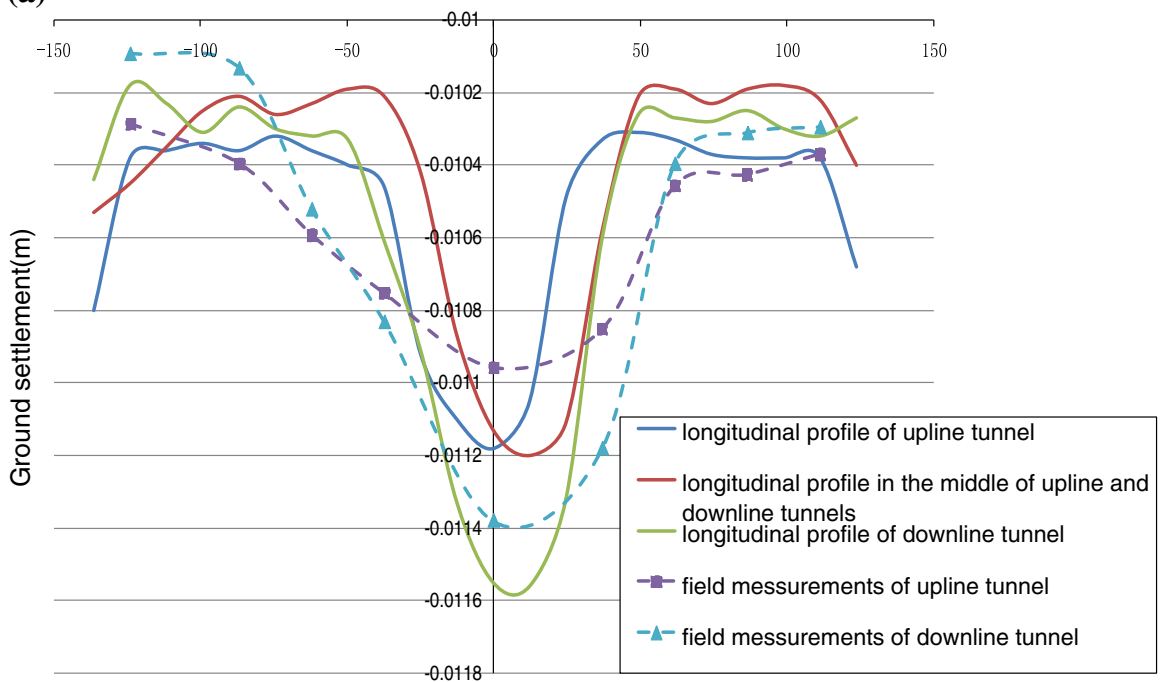

(b) The distance between ground point and cross point $(\mathrm{m})$

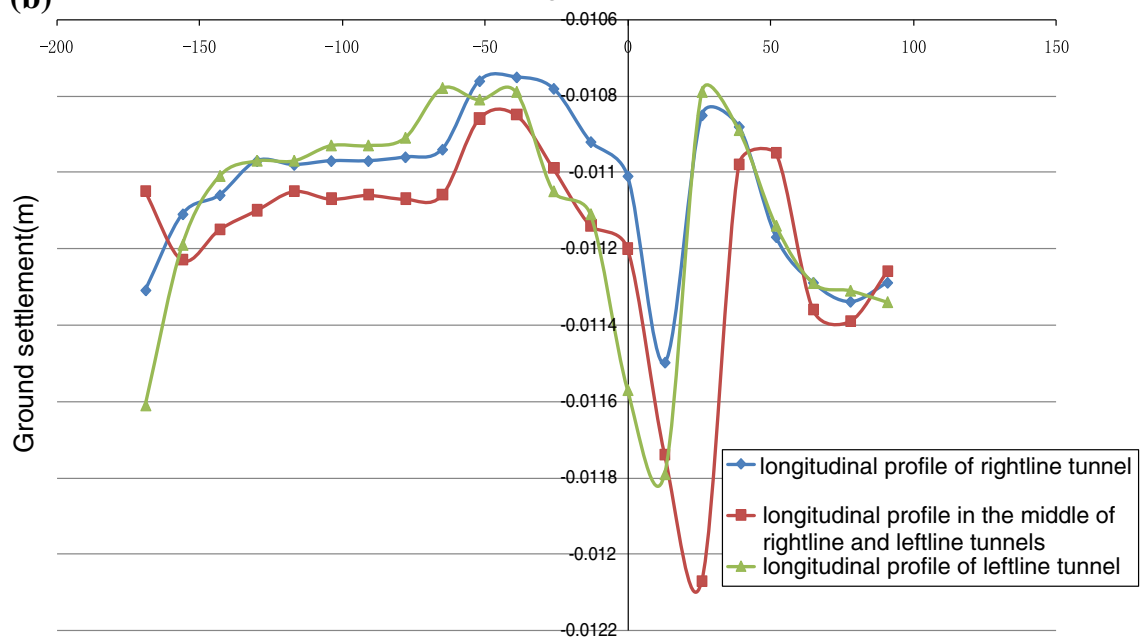

Fig. 13 Ground settlement curves of the overlapped tunnels engineering. a Ground settlement curve and data of longitudinal profile of metro No.1 line-0.0122, b ground settlement curve of longitudinal profile of Centralized transportation system

Table 4 Comparative analysis of the results

\begin{tabular}{llll}
\hline Category & Criterion & Discriminant parameter & State discrimination \\
\hline $\begin{array}{l}\text { Energy evolution model } \\
\text { (Eq. } 17 \text { and Table 1) }\end{array}$ & Energy evolution degree & $4.0000-4.0761$ & Stable \\
$\begin{array}{l}\text { Energy evolution criterion } \\
\text { [Eq. } 18 \text { and criterion (1) } \\
(2),(3)]\end{array}$ & Correlation dimension of final state $\mathrm{D}_{2}$ & $<2$ & Stable \\
& Variation tendency of correlation dimension $\mathrm{D}_{2}$ & $\begin{array}{c}\text { Increasing-dimension in } \\
\text { small interval }\end{array}$ & Stable \\
& Entropy $K_{2}$ in final state & Close to 0 & Stable \\
& Variation tendency of Entropy $K_{2}$ & Increase first and then decrease & Stable \\
Finite element model & Designed settlement standard: +10 to $-30 \mathrm{~mm}$ & +9.21 to $-22.62 \mathrm{~mm}$ & Stable \\
Actual monitoring & Settlement warning value: $\pm 20 \mathrm{~mm}$ & +9.21 to $-16.29 \mathrm{~mm}$ & Stable
\end{tabular}


project is hoped to provide reference for discrimination ideas and methods of tunnel stability under the complex engineering conditions. And it will take some time to collect information and dassta of other projects instability. The new applications of model and criterion will also further develop in future studies and will be published in time.

\section{Conclusion}

1. Based on situ measurements, the thermodynamics laws and the dissipative structure theory, external incremental work, energy and incremental dissipation are studied for tunnel system instability in operation and management. And the energy evolution model of tunnel system is established in this paper. On this basis, the energy criterion of the evolution process of stability with large $(\geq 3,000)$ and small $(<3,000)$ sample sizes is confirmed.

2. The stability in an interchange tunnel for Line 1 in the Guangzhou metro and the Pearl River New Town centralized transportation system at Tianhenanyilu is analyzed, and compared with the monitored results. It is revealed that the undercrossing of the centralized transportation system has little influence on Line 1, which has been always been stable. Meanwhile, the reliability of a dynamic evolution model and failure criterion for the dynamic instability of tunnel systems established in the paper is verified, which has reference value on the discriminant of tunnel stability in the actual project.

3. Compared with the traditional numerical simulation and early warning by using fitting monitored data, the research in this paper regards the tunnel as a system, which quantitatively reveals its evolution mechanism of the energy dissipation process under the combined action of comprehensive factors inside and outside from the perspective of dynamic mechanism, and put forward a discriminate method in different evolutionary stages and instable critical state, which provide new ideas and methods in tunnel engineering risk awareness and control.

Acknowledgments This research is funded by the following grants: (1) Project of the State Key Program of National Natural
Science of China (Grant No. 41030747, No. 41102181); (2) Project of the Guangdong Provincial Science and Technology Program (Grant No. 2010A030200007, No. S2011010002 104, No. S2011020001229) (3) Project of the Guangzhou Science and Technology Program (No. 2010GN-D00011, No. 2013J2200075).

Open Access This article is distributed under the terms of the Creative Commons Attribution License which permits any use, distribution, and reproduction in any medium, provided the original author(s) and the source are credited.

\section{References}

Aydan Ö, Uehara F, Kawamoto T (2012) Numerical study of the long-term performance of an underground powerhouse subjected to varying initial stress states, cyclic ssswater heads, and temperature variations. Int J Geomech 12(1):14-26

Bhasin R, Magnussen AW, Grimstad E (2006) The effect of tunnel size on stability problems in rock masses. Tunn Undergr Space Technol 21(3):405-406

Cai SH (1998) Dissipative structure with non-equilibrium phase transition theory and application. Guizhou Science and Technology Press, Guiyang

Carranza Torres C (2004) Elasto-plastic solution of tunnel problems using the generalized form of the Hoek-Brown failure criterion. Int J Rock Mech Min Sci 41(3):480-481

Chen XG (2002) Study on failure and criteria of tunnel structure. Doctoral thesis of Southwest Jiaotong University, Chengdu

Cui XZ (2010) Dynamic numerical analysis of antimoisturedamage mechanism of permeable pavement base. Int $\mathrm{J}$ Geomech 10(6):230-235

Das Sarat Kumar, Basudhar Prabir Kumar (2011) Parameter optimization of rock failure criterion using error-in-variables approach. Int J Geomech 11(1):36-43

Gong LH (2005) The study of Kolmogorov entropy of the dynamic system. J Daxian Teach Coll (Natural Science Edition) 15(5):15-19

Haining Cui (2009) The theory of thermodynamic systems. Jilin University Press, Changchun

Heap MJ, Faulkner DR (2008) Quantifying the evolution of static elastic properties as crystalline rock approaches failure. Int J Rock Mech Min Sci 45:564-573

Hong Jiang (2013) Deep buried tunnel surrounding rock stability analysis of the cusp catastrophe theory. J Chem Pharm Res 5(9):507-514

Huang PT, Hu LY (2004) To clarify the concept of negative entropy, information entropy and the entropy principle. Mod Phys 16(3):21-23

Huang F, Yang XL (2011) Upper bound limit analysis of collapse shape for circular tunnel subjected to pore pressure based on the Hoek-Brown failure criterion. Tunn Undergr Space Technol 26:614-618

Jiancong Xu (2008) Research on grey-cusp-catastrophic destabilization prediction model of tunnel surrounding rock and primary support system. J Rock Mech Eng 27(6):118-1181

Komiya K, Shimizu E, Watanabe T et al (2000) Earth pressure exerted on tunnels due to the subsidence of sandy ground. In: 
Proceedings of Geotechnical Aspect of Underground Construction in Soft Ground. Rotterdam: A. A. Balkema 397-402

Li SH (1998) Study of stability of surrounding rocks of tunnels from cybernetic points of view and its application. J China Coal Soc 2:23-29

Liu GY, Liu XX, Wei XY (2002) A cusp catastrophic model for tunnel caving. J Geol Hazard Environ Preserv 13(2):59-62

Liu HY, Small JC, Carter JP (2008) Full 3D modelling for effects of tunnelling on existing support systems in the Sydney region. Tunn Undergr Space Technol 23:399-420

Lu JH, Lu JA, Chen TH (2005) Chaotic time series analysis and its applications. Wuhan University Press, Wuhan

Serrano A, Olalla C, Reig I (2011) Convergence of circular tunnels in elastoplastic rock masses with non-linear failure criteria and non-associated flow laws. Int J Rock Mech Min Sci 48:878-887

Shen W (2001) The theory of self-organization and dissipative structures and its application in geology. Geology-geochemistry 29(3):1-7

The second project department of Centralized transportation system in municipal transportation project area of $\mathrm{Gu}$ angzhou Pearl River new town of China Railway Tunnel Group (2007) The second section of Centralized transportation system in municipal transportation project area of Guangzhou Pearl River new town: The construction plan of shield passing through operating metro NO.1 lineR (JJJZ002). Technical report

Wang XM (2005) From entropy and information entropy to selforganizing. Mod Phys 15(5):15-19
Wang PL, Song B, Wang L (2006) Study on Kolmogorov entropy based on chaotic time series. Comput Eng Appl 21:162-164

Wenjiang Li, Sumin Zhang, Xianmin Han (2011) Judgement method for surrounding rock stability of guanjiao tunnel based on catastrophe theory. Appl Mech Mater 90-93:2307-2312

Yan CB, Xu GY (2006) Instability analysis of deep-buried tunnels with catastrophe theory. J Eng Geol 14(4):508-512

Yang BL (1996) Basic concepts research of classical thermodynamics. Science press, Beijing

Yang XL, Huang F (2011) Collapse mechanism of shallow tunnel based on nonlinear Hoek-Brown failure criterion. Tunneling and Underground Space Technology 26:686-691

Yang F, Yang JS (2010) Stability of shallow tunnel using rigid blocks and finite-element upper bound solutions. Int J Geomech 10(6):242-247

Yang XL, Yin JH (2006) Linear Mohr-Coulomb strength parameters from the non-linear Hoek-Brown rock masses. Int J Rock Mech Min Sci 41:1000-1005

Yue Pan, Yong Zhang, Guangming Yu (2006) Mechanism and catastrophe theory analysis of circular tunnel rockburst. Appl Math Mech Heidelb 27(6):841-852

Yuheng Hu (1989) Systems theory, information theory, cybernetics principle and their application. Henan Press, Zhengzhou

Zhang SM, Liu Y (2003) Simulation of limit displacements for tunnel upper bench. J Shijiazhuang Railw Inst 16(Supp. 1):2-7 